\title{
PROBLEMS OF ASSESSING SPORTS FITNESS BY MULTIVARIATE TEST BATTERIES
}

\section{THOMAS \\ Liverpool Polytechnic}

\section{INTRODUCTION}

Sports fitness is defined for the purpose of this paper as 'the capacity of an individual to perform a specific sporting function at a given time'. In this case, participation in sport constitutes an assessment of sports fitness. If the competitor does nothing other than compete, then there is no need to assess his fitness outside the competition unless there are pre-competitive medical requirements such as in boxing, parachuting etc.

If the competitor is involved in preparation for competition which is designed to improve his fitness (defined as training) he is in a control system. The input to the system is his potential for fitness and his training. The output is his new level of fitness. Optimum control of the training system can only be achieved with feedback of output information, which can be monitored in two ways.

The competitive performance, which is an amalgam of a very great number of variables and subject to many extraneous influences, may be used. It is probable that the majority of fitness assessments are of this type.

Secondly, a fitness assessment may be performed which is different from the competitive performance. This may rarely be achieved during the performance.

The only activity in which all the performance variables may be monitored simultaneously is the competition itself. The most valid single assessment of sporting fitness is sporting performance; but that performance may conceal a wide variety of both critical and non critical factors, and that assessment may provide inadequate information upon which to base alterations in the control system. Additionally, competitive performance (especially maximal performance) may occur at infrequent intervals, in which case critical lag may occur in the control system.

It is apparent that a multiplicity of fitness variables needs to be monitored, at sufficiently frequent intervals to obtain optimal feedback information, and of at least those variables which are critical to the competitive performance.

\section{VARIABLES}

The present state of sports science allows few unequivocal statements to be made concerning those fitness variables which are absolutely critical to a specific sporting function. Maximal oxygen uptake tends to be accepted in a wide variety of locomotor performances but even this physiological index is always subject to supplementation by an individual's potential for anaerobic function. For the purpose of training, it is more productive to consider variables which are highly (if not absolutely) critical to performance. The selection of these variables requires a process of validation, generally achieved by a correlation of the variable in question against the performance itself, or against representative components of the performance.

Whilst it may not be possible to select absolutely critical single variables, especially since the importance of any one may be dependent on the state of one or more others it seems worthwhile to search in a matrix of variables for groups of two or more which together achieve higher correlations with performance, or which account for the major part of the variance present in the total output of information. These may be termed 'principal components' of at least those fitness variables which are being monitored. The process of development of multivariate assessment of sports fitness involves continuous rejection of less useful variables, trials of new variables, tests of validity against performance, and identification of principle components. The potential for measurement is very great, and may be classified into environmental, anthropometrical, physiological, sociological and psychological factors. The reliability of measurement varies greatly between and within these categories. It is counterproductive to reject tests of low reliability unless more reliable tests are available.

\section{TEST BATTERIES}

The major problems of test batteries are validity and objectivity, logistic feasibility and frequency. Essentially, each competitor is unique in the relationship between his performance variables - principal components established for groups may not be relevant for individuals within the group. Homogeneity is largely absent from groups of competitors, even when they all perform similar sporting functions. It follows that test batteries ought ideally to be specific to the individual, less ideally specific to the group, and least ideally across the total sports population IN TERMS OF OPTIMISING TRAINING CONTROL.

On the other hand, the paucity of definitive information concerning relevance of tests to functions indicates that sports science is not yet in a position to specify test batteries for individuals or specific sports. The use of general test batteries across the whole population will permit a gradual refinement of constituent tests, and a coalescence of specific test batteries in the first instance for specific sports, and finally for each individual competitor. 